\title{
The Relationship Between Mindfulness, Depressive Symptoms, and Non-Suicidal Self- Injury Amongst Adolescents
}

\begin{abstract}
\end{abstract}
Mindfulness is often part of treatment for non-suicidal self-injury (NSSI); however, there has been limited research examining the role of mindfulness in NSSI. Thus, the current study sought to investigate the relationship among mindfulness, depressive symptoms, and NSSI (past year) in adolescents ( $N=764 ; 56.8 \%$ female, $M_{\text {age }}$ $=14.42, S D=0.64)$ with consideration of gender. Adolescents with recent NSSI $(n=74$; $83.8 \%$ female, $\left.M_{\text {age }}=14.36, S D=0.56\right)$ and a matched for age and gender no-NSSI group completed measures of mindfulness and depression. Findings revealed that mindfulness and depressive symptoms were negatively correlated, although significantly less so for the NSSI group. Second, the NSSI group reported greater depressive symptoms and less mindfulness. Finally, mindfulness was found to partially mediate the effect of depressive symptoms on NSSI. The present study is the first to provide empirical support for the protective role of mindfulness in NSSI.

Non-suicidal self-injury (NSSI) is defined as the deliberate, self-inflicted destruction of body tissue without suicidal intent, and for purposes not socially sanctioned (Heath \& Nixon, 2009). The majority of individuals begin self-injuring in early adolescence, between the ages of 13 and 15 years (Heath, Toste, Nedecheva, \& Charlebois, 2008; Rodham \& Hawton, 2009). In addition, adolescents are the most highrisk group, with 15 to $20 \%$ of community-sample adolescents reporting self-injuring at least once in their lives (e.g., Muehlenkamp \& Gutierrez, 2007; Ross \& Heath, 2002), and one quarter of these adolescents self-inuring repetitively (Laye-Gindhu \& Schonert- 
Reichl, 2005; Whitlock, Powers, \& Eckenrode, 2006). The high prevalence of NSSI among non-clinical samples of adolescents is of concern as NSSI is associated with a variety of mental health difficulties (Plener, Schumacher, Munz, \& Groschwitz, 2015). A substantial amount of this literature focuses on NSSI in the context of Borderline Personality Disorder (BPD; Bracken-Minor \& McDevitt-Murphy, 2014; Brickman, Ammerman, Look, Berman, \& McCloskey, 2014; Sadeh et al., 2014). Although the link between NSSI and BPD has been consistently documented in clinical samples, recently the association between NSSI and depression has been highlighted (see Plener et al., 2015 for review). Specifically, depressive symptoms have been shown to be positively correlated with NSSI (Hankin \& Abela, 2011; Muehlenkamp \& Gutierrez, 2007) and predictive of NSSI in community samples of adolescents (Marshall, Tilton-Weaver, \& Stattin, 2013). Taking into account this newly emerged link with depression, and given the lack of evidence-based treatment and prevention options for NSSI in adolescents (Nock, 2012), an investigation of the established protective factors for depressive symptoms is an important area of research for individuals who report engaging in NSSI.

Mindfulness-based techniques are an increasingly popular form of intervention for an array of mental health disorders, as well as the optimization of well-being (e.g., Beauchemin, Hutchins, \& Patterson, 2008; Burke, 2010; Kabat-Zinn, 2003; Mendelson et al., 2010; Tacon, McComb, Caldera, \& Randolph, 2003). Mindfulness is a nonjudgmental deep and focused awareness and acceptance of the present moment that is typically experienced through the practice of meditative techniques (Kabat-Zinn, 2003). A variety of reviews support the efficacy of mindfulness-based techniques to reduce depressive relapse (Teasdale et al., 2002) and anxiety symptoms in adults (Grossman, Niemann, 
Schmidt, \& Walach, 2004) and children (Semple, Reid, \& Miller, 2005). Furthermore, results from a meta-analysis suggest that mindfulness-based techniques may address processes that occur in multiple disorders such as anxiety and depressive symptoms (Hofmann, Sawyer, Witt, \& Oh, 2010). Thus, the use of mindfulness techniques is supported across a range of psychological problems.

Recently, enhancing mindfulness has been suggested as a beneficial approach to help adolescents who self-injure (Garisch \& Wilson, 2015; Lundh, Karim, \& Quilisch, 2007). For instance, mindfulness is an important element of the intervention used in an adaptation of Dialectical Behavior Therapy (DBT; Linehan et al., 2006) for adolescents (Miller, 1999; Wagner, Rathus, \& Miller, 2006). There is some evidence that this adapted intervention may reduce self-injury and improve psychosocial adjustment among adolescents who self-injure (Katz, Cox, Gunasekara, \& Miller, 2004; Miller, Wyman, Huppert, Glassman, \& Rathus, 2000; Rathus \& Miller, 2002). It is not yet known whether associated changes in the participants' experienced mindfulness are a significant contributor to these observed benefits; although, the aforementioned research suggests the need for future examination of the relationship of mindfulness and self-injury.

In addition to experiencing a state of mindfulness in response to an intervention or event, individuals may differ in their general tendency to be mindful day-to-day. Specifically, beyond a mindfulness state, mindfulness can also occur as an individual disposition. Dispositional mindfulness refers to the mindfulness experienced during daily activities (Brown \& Ryan, 2003; Brown, Ryan, \& Creswell, 2007; Kabat-Zinn, 2003; Murphy, Mermelstein, Edwards, \& Gidycz, 2012). Variations in dispositional mindfulness may occur naturally or may be developed through an intervention and/or 
engagement in regular mindfulness practice (Brown \& Ryan, 2003). In adults, dispositional mindfulness has been found to be associated with lower levels of reported stress, anxiety, depression (Brown \& Ryan, 2003; Cash \& Whittingham, 2010; Segal, Williams, \& Teasdale, 2002), and negative affect (Arch \& Craske, 2010), and positively correlated with perceived health (Stillman, Feldman, Wambach, Howard, \& Howard, 2014) and positive states of mind (Branstrom, Duncan, \& Moskowitz, 2011). Although there is limited research examining dispositional mindfulness in adolescence (Ciesla, Reilly, Dickson, Emanuel, \& Updegraff, 2012; Marks, Sobanski, \& Hine, 2010), there is emerging evidence that dispositional mindfulness in adolescents is associated with lower reports of social anxiety, and higher reports of self-esteem (Rasmussen \& Pidgeon, 2011).

In a recent review of the state of the field for mindfulness in youth (Greenberg \& Harris, 2012), it was concluded that although there is sufficient evidence of the benefits of mindfulness in adults, the benefits of mindfulness for youth remain promising but as yet unsubstantiated (Burke, 2010; Frank, Jennings, \& Greenberg, 2013; Greenberg \& Harris, 2012). Thus, there is currently a need for further study of possible benefits of mindfulness in adolescence in general. Furthermore, despite the inclusion of mindfulness in most treatments for NSSI (e.g., Gratz, 2007; Miller, Muehlenkamp, \& Jacobson, 2009; Muehlenkamp, 2006), the direct study of mindfulness in adults or adolescents who selfinjure is surprisingly limited.

However, in a related area, Wupperman, Fickling, Klemanski, Berking, and Whitman (2013) examined whether a lack of mindfulness mediated the relationship between BPD features, self-injury (defined as “including cutting, burning, scratching, 
etc." p. 906), and other harmful dysregulated behaviors (defined as "including substance misuses, binge eating, aggression, etc." p. 906) in a sample of 70 adult psychiatric inpatients (84.2\% female) with current critical impairment (e.g., suicidal ideation, destructive behaviors) and past psychological trauma. Participants completed the Mindfulness Attention Awareness Scale (MAAS), the Borderline Personality Features Scale of the Personality Assessment Inventory (PAI-BOR), and an adapted Means-End Problem-Solving Test for emotions (MEPS-Emo) to assess self-injury and dysregulated behaviors. Results revealed a significant negative correlation between mindfulness and BPD features, acts of self-injury, and acts of harmful dysregulated behavior.

Furthermore, deficits in mindfulness mediated the relationship between BPD features and acts of self-injury and acts of harmful behavior. These findings suggest that mindfulness may serve as a protective factor for self-injury in individuals with BPD; however, since this study was limited to a sample of adults with BPD and used a non-specific definition of self-injury, it would be important to examine whether this relationship also exists for NSSI in non-BPD samples of adults and adolescents.

Research has begun to examine the association between mindfulness and deliberate self-harm (DSH; a broader term which does not distinguish whether suicidal intent is present or not and includes self-poisoning and substance abuse; Haw, Houston, Townsend, \& Hawton, 2002) in adults and adolescents. Amongst adults, a 2014 study examining the relationship between dispositional mindfulness, self-control, aggression, and DSH in 241 adults (63.07\% female), found mindfulness to be positively correlated with self-control and negatively correlated with aggression and self-harm (Yusainy \& Lawrence, 2014). Individuals who engaged in self-harm were lower in mindfulness, 
although they were also found to be lower in self-control. In their examination of whether mindfulness is predictive of self-harm, the authors showed that self-control mediated the relationship between mindfulness and self-harm. This study suggests that mindfulness is strongly related to self-control; thus, mindfulness may serve a protective role in terms of the engagement in self-harm through its influence on self-control. However, in this study, the authors did not examine NSSI specifically, and although they did find that mindfulness through aspects of self-control was predictive of the presence or absence of self-harm, they did not take into account the determinants of mindfulness as a protective role in the presence of difficult emotions such as depressive symptoms.

In a study with adolescents, Lundh et al. (2007) examined the association between deliberate self-harm and mindfulness in 123 Swedish adolescents (39.02\% female) using the Deliberate Self-Harm Inventory and the MAAS. Results revealed that youth reporting self-harm $(n=51)$ indicated lower levels of mindfulness compared to participants who did not engage in deliberate self-harm $(n=72)$. However, the inclusion of both suicidal and non-suicidal self-injury may be misleading as mindfulness may vary in those with suicidal behaviors.

Very recently, in a study examining correlates of NSSI in a community sample of adolescents at two time points (Time 1: $n=1162,43 \%$ female; Time $2: n=830,47 \%$ female), NSSI was found to be negatively correlated with mindfulness as measured by the Revised Cognitive and Affective Mindfulness Scale (CAMS-R; Garisch \& Wilson, 2015). Specifically, mindfulness was significantly negatively associated with reports of both current (past 3-8 months) and lifetime history of NSSI. Furthermore, higher mindfulness at Time 1 was associated with lower engagement in NSSI at Time 2, which 
further supports the notion that mindfulness may serve as a protective factor for NSSI engagement.

In summary, mindfulness is generally recommended in treating depressive symptoms in adolescents (e.g., Marks et al., 2010), with depression being found to be a strong predictor of NSSI (Plener et al., 2015) and yet the relationship between mindfulness and depressive symptoms in those who self-injure remains unexplored. Despite the evidence that lower dispositional mindfulness is associated with reports of non-specific self-injury in clinical populations (Wupperman et al., 2013), deliberate selfharm in adolescents and adults (e.g., Lundh et al., 2007; Yusainy \& Lawrence, 2014), and NSSI in adolescents (Garisch \& Wilson, 2015), no study to date has examined the complex relationship among dispositional mindfulness, depressive symptoms, and nonsuicidal self-injury among adolescents.

Thus, the objectives of the current study were to (1) examine the relationship among depressive symptoms, mindfulness, and gender in youth who report engaging in NSSI in the past year (NSSI group) and a no-NSSI comparison group, (2) to investigate possible NSSI/no-NSSI group and gender differences in depressive symptoms and mindfulness, and (3) to evaluate the role of mindfulness as a possible mediator between depressive symptoms and NSSI. Hypotheses corresponding to these objectives are as follows. It was hypothesized, H1, there would be a significant negative correlation between depressive symptoms and mindfulness for both NSSI and no-NSSI groups; H2 that the NSSI group would report significantly more depressive symptoms than the noNSSI group and H3 less mindfulness than their non-self-injuring peers; and finally, (H4) that mindfulness would mediate the relationship between depressive symptoms and 
NSSI. No specific hypotheses were made regarding gender differences as these were exploratory in nature.

\section{Method}

\section{Participants and Procedure}

Grade 9 students $\left(N=764 ; 56.8 \%\right.$ female, $\left.M_{\text {age }}=14.42, S D=0.64\right)$ were recruited from 17 high schools for a study on stress and coping during adjustment to high school. Students were provided with both an information letter about the project as well as consent forms for their parents. Students who had parental consent and were willing to participate in the study were asked to fill out a battery of measures in their school cafeteria, where black cardboard separators were provided in order to preserve their confidentiality. All students who completed the questionnaires were entered in a raffle to win one of four $50 \$$ cinema gift cards. Since participants who screened positive for NSSI participated in subsequent interviews to confirm NSSI, a $\$ 10$ gift card was given for the second interview.

Of the 764 students who participated, 159 participants were excluded from analyses either because (a) they did not complete all the measures or indicate a gender ( $n$ $=113)$, (b) they were identified as being univariate or multivariate outliers $(n=21)$, or (c) their history of NSSI could not be confirmed $(n=25)$; thus creating a final pool of 605 participants $\left(57 \%\right.$ female, $\left.M_{\text {age }}=14.41, S D=0.67\right)$. Of these, $17.97 \%$ of females $(n=62)$ and $4.62 \%$ of males $(n=12)$ reported engaging in NSSI over the past 12 months, comprising the final NSSI group of 74 participants ( $83.8 \%$ female, $M_{\text {age }}=14.36, S D=$ 0.56). Of these 74 participants who indicted engagement in NSSI, 67 responded to the 
question concerning age of onset; $23.9 \%$ stated they first engaged in NSSI before the age of $12,44.8 \%$ indicated an age of onset of 12 or 13 , and $31.3 \%$ reported an age of onset of 14 or 15 . Reported lifetime frequency of NSSI incidents were as follows: one time $5.8 \%$, 2-4 times $27.5 \%, 5-10$ times $26.1 \%, 11-50$ times $17.4 \%, 51-100$ times $18.8 \%$ and over 100 times 4.3\%. Most common methods endorsed were cutting (65.8\%) and scratching (49.3\%), followed by banging to the point of bruising (35.6\%). Finally, participants' reports of emotion following the self-injury revealed most frequently endorsed feelings

were ashamed (43.8\%), guilty (35.6\%), sad (35.6\%), scared $(31.5 \%)$, and angry $(31.5 \%)$ followed by calm (28.8\%) and nervous $(28.8 \%)$.

A gender-matched comparison group was then created using SPSS v.22 by randomly selecting from the larger pool of participants not engaging in NSSI $(n=497)$ a number of males and females equal to the number of males and females engaging in NSSI. Therefore, the final sample consisted of 148 Grade 9 students $\left(83.8 \%\right.$ female; $M_{\text {age }}$ $=14.36, S D=0.522$ ). This project was approved by the university Research Ethics Board.

\section{Measures}

The How I Deal with Stress Questionnaire (HIDS; Heath \& Ross, 2007), a selfreport self-injury screening measure for use in community settings, was used to screen and assess for NSSI. The first section is made up of 32 items assessing the frequency of use of various healthy (e.g., "talk to someone", "listen to music") and unhealthy (e.g., “drink alcohol", "hit someone") coping behaviors on a 4-point Likert scale ranging from never (0) to always (3). One of these items ("physically hurt myself on purpose") assesses engagement in self-injury as a strategy to cope with stress. Participants also 
complete a follow-up section of the HIDS which further asks the participant "Have you engaged in the following self-injury behaviors on purpose and without wanting to die?" If the participant indicates "yes" at this point, they are asked to report on the method they used, their emotional state after engaging in NSSI, the frequency of the behavior, and the age of onset. Participants are asked to fill out this part in order to ensure the reported behavior is consistent with the definition of NSSI. The HIDS has successfully been used in community settings to identify engagement in NSSI in adolescents (Ross \& Heath, 2002). Moreover, Toste, Christie, and Heath (2011) have found high test-retest reliability for the NSSI screening item $(r=.83)$ after 6 months.

NSSI categorization was based on whether participants who completed the HIDS reported having physically hurt themselves on purpose without suicidal intent over the past 12 months and identified specific behaviors consistent with the definition of NSSI. NSSI engagement was further confirmed using the Self-Injurious Thoughts and Behaviors Interview (SITBI; Nock, Holmberg, Photos, \& Michel, 2007), which is a structured interview assessing five types of self-injurious thoughts and behaviors (suicidal ideation, suicide plans, suicide gestures, suicide attempts, and NSSI). Questions in the NSSI section of the SITBI assess the frequency of NSSI engagement, the types of methods used, as well as the onset and continuity of the behaviors. Nock et al. (2007) found that the NSSI module had strong test-retest reliability (average $\kappa=.70$, intraclass correlation coefficient $=.44$ ) after 6 months and that there was good agreement between the SITBI and the Schedule for Affective Disorders and Schizophrenia for School Aged Children - Present and Lifetime Version (K-SADS-PL; Kaufman et al., 1997) on the presence of NSSI $(\kappa=.74)$ and between the SITBI and the Functional Assessment of 
Self-Mutilation (FASM; Lloyd, Kelley, \& Hope, 1997) on the presence $(\kappa=1.0)$ and lifetime frequency of NSSI $(r=.99)$.

Mindfulness was assessed using the Child and Adolescent Mindfulness Measure (CAMM; Greco, Baer, \& Smith, 2011), a self-report questionnaire used to assess dispositional mindfulness. It consists of 10 reverse-scored items rated on a 5-point Likert scale ranging from never (0) to always true (4). The CAMM specifically assesses both present moment awareness (e.g., "At school, I walk from class to class without noticing what I'm doing”) and non-judgmental acceptance of thoughts and feelings (e.g., "I get upset with myself for having certain thoughts"). It has been found to have good divergent validity with measures of thought suppression $(r=-.58, p<.01)$ and psychological inflexibility $(r=-.60, p<.01)$ when used with a sample of children and adolescents (Greco et al., 2011). In the current sample, the CAMM demonstrated a high internal consistency $(\alpha=.88)$.

Depressive symptoms were assessed using the Beck Depression Inventory for youth (BDI-Y; Beck, Beck, Jolly, \& Steer, 2005), which consists of 20 items on a 4-point Likert scale ranging from never (0) to always (3). The BDI-Y has good internal consistency, with coefficient alphas ranging from .90 to .95 depending on age group and gender in a sample of children and adolescents ranging from 7 to 18 years of age and high test-retest reliability after a 1 week period ( $r>.74$ for all age groups) (Beck et al., 2005). Beck et al. (2005) also found that the BDI-Y has good convergent validity, as shown by being positively correlated with the Children's Depression Inventory $(r=.67$ in a sample of adolescents). The present study converted participants' raw scores into standardized scores based on gender and age. 


\section{Data Analysis}

Pearson and biserial correlation coefficients with Bonferonni corrections were conducted to test the hypothesized associations between mindfulness and depressive symptoms for both groups (H1). Two 2 (NSSI; no-NSSI) X 2 (Male; Female) analyses of variance (ANOVAs) were conducted to assess for the expected differences in reports of depression (H2) and mindfulness (H3). Finally, the Preacher and Hayes (2004) bootstrapping procedure with 5,000 resamples was used to test the mediational role of mindfulness (H4). This method of analysis has the advantage of not being based on largesample theory nor does it rely on assumptions of the shape of the distribution decreasing the chances for Type I and Type II errors.

\section{RESULTS}

\section{Objective 1}

An initial objective of the present study was to assess the relationship between mindfulness, depressive symptoms, and gender within the NSSI group and the no-NSSI group. Table 1 presents the calculated correlation coefficients. Results support the hypothesized associations; however, the correlation between mindfulness and depressive symptoms was significantly lower in the NSSI group as confirmed by a Fisher's r-to-z transformation $(z=-2.16, p=.03$, two-tailed). Scatterplots to illustrate the differences in the relationship between mindfulness and depressive symptoms in the NSSI and no-NSSI group (Figure 1), reveal that in the NSSI group there is a small subset of adolescents who report both high mindfulness and high depressive symptoms. In addition, with respect to gender, the biserial correlations did not show NSSI/no-NSSI group differences in the 
relationship between gender and mindfulness, although the relationship between gender and depressive symptoms was only significant in the no-NSSI group, with females reporting higher levels of depressive symptoms than males.

\section{Objective 2}

The second objective was to investigate possible NSSI/no-NSSI group and gender differences in depressive symptoms and mindfulness. Table 2 displays the mean and standard deviations for depressive symptoms and mindfulness, by gender and NSSI group. It was hypothesized (H2) that the NSSI group would report greater depressive symptoms than the no-NSSI group. After the full factorial model revealed the interaction between NSSI Groups and Gender was not significant for reports of depressive symptoms $\left(F(1,147)=0.83, p=.37 . \eta^{2}=.01\right)$ and mindfulness $\left(F(1,147)=0.58, p=.45, \eta^{2}=.00\right)$, the $2 \times 2$ ANOVAs were rerun as main effects models. Results of a 2 (NSSI; no-NSSI) X 2 (Male; Female) ANOVA using a main effects model supports this hypothesis, revealing a NSSI/no-NSSI group effect in depressive symptoms reported $(F(1,147)=74.21, p<$ $\left..001, \eta^{2}=.34\right)$, with the NSSI group reporting significantly higher levels of depressive symptoms. The main effect for gender was not significant $\left(F(1,147)=1.59, p=.21, \eta^{2}=\right.$ $.01)$. It was further hypothesized that the NSSI group would report lower mindfulness than the no-NSSI group (H3). The results of a $2 \times 2$ ANOVA using a main effects model support this hypothesis, showing a NSSI/no-NSSI group effect $(F(1,147)=43.20, p<$ $\left..001, \eta^{2}=.23\right)$ with the NSSI group reporting significantly lower mindfulness than their no-NSSI peers. The main effect for gender was not significant $(F(1,147)=1.05, p=.31$, $\left.\eta^{2}=.01\right)$.

\section{Objective 3}


The final objective was to test the (H4) hypothesized model of mindfulness as a mediator in the relationship between depressive symptoms and NSSI. The Preacher and Hayes' (2004) PROCESS application for SPSS was used to test this model. As shown in

Figure 2, the results suggest reports of greater depressive symptoms were associated with lower mindfulness (path a: $-0.35 ; 95 \%$ CI $[-0.43,-0.26]$ ). Reports of lower mindfulness were additionally associated with NSSI Group membership (path $b$ : OR $=0.93 ; 95 \%$ CI $[-0.88,0.99])$. The total effect of depressive symptoms on NSSI groups (path $c$ : OR = $1.15 ; 95 \%$ CI $[1.06,1.17])$ suggests greater depressive symptoms were predictive of NSSI Group membership. When controlling for mindfulness, the direct effect of depressive symptoms on NSSI groups lowered (path c': OR $=1.12 ; 95 \%$ CI $[1.01,1.05]$ ) but continued to be significant, suggesting mindfulness may act as a partial mediator. An analysis of the mediational role of mindfulness within this model revealed a positive indirect effect $(\mathrm{OR}=1.02 ; 95 \% \mathrm{CI}[1.00,1.05])$, supporting the overall hypothesis that mindfulness mediates the relation between depressive symptoms and NSSI grouping. Further support for this hypothesis was provided by conducting a Sobel test $(z=2.04, p=$ $.04)$.

\section{DISCUSSION}

Despite the common practice of seeking to enhance mindfulness as part of treatment in youth who self-injure (e.g., Miller et al., 2009; Rathus \& Miller, 2002; Walsh, 2012), to the authors' knowledge, the present study is the first to investigate dispositional mindfulness in youth who report past year engagement in NSSI and the only study to evaluate the relationship of mindfulness and depressive symptoms in youth who engage in NSSI. 
As expected, there was a significant negative correlation between mindfulness and depressive symptoms for both the NSSI and no-NSSI group, which is consistent with Marks et al. (2010) findings as well as numerous studies documenting the negative association between reports of mindfulness and depression, anxiety and/or stress in adults (Brown \& Ryan, 2003; Cash \& Whittingham, 2010; Segal et al., 2002) and adolescents (Rasmussen \& Pidgeon, 2011). Interestingly, the negative correlation between depressive symptoms and mindfulness reports was significantly lower for the NSSI group than their non-self-injuring peers. Visual examination of the scatter plots revealed that although for the NSSI group there is a pattern of increasing mindfulness being associated with lower depressive symptoms similar to the no-NSSI group, there is also a small subset of NSSI youth reporting both high depressive symptoms and high mindfulness. This finding was unexpected and may reflect the fact that for some adolescents with more severe depression, mindfulness may be experienced as an increased focus on present moment suffering. Possibly, although mindfulness and rumination are generally inversely related, with increases in mindfulness being associated with concomitant decreases in rumination (Ciesla et al., 2012; Marks et al., 2010), in certain cases for those with severe depression or anxiety, specific facets of mindfulness (e.g., present moment awareness) may increase focus on negative thoughts and sensations. Although requiring replication and tentative at this time, this is an important finding as it suggests that for youth with NSSI who report high levels of depressive symptoms, treatment that seeks to enhance mindfulness should do so cautiously, and the experience of mindfulness by the youth should be monitored. Most interventions for youth who self-injure include some mindfulness training (e.g., Miller et al., 2009; Rathus \& Miller, 2002; Walsh, 2014), but these typically seek to 
support the youth in experiencing a mindfulness state (i.e., focus on the present moment with acceptance and without judgement) with the guidance and supervision of the mental health professional. This type of mindfulness experience may be very different than in the present study, in which youth were asked about their day-to-day tendency to experience mindfulness, or dispositional rather than state mindfulness. Nevertheless, mindfulness programs are increasingly being universally offered in schools (e.g., Burke, 2010; Mendelson et al., 2010) with little monitoring or understanding of the possible contraindications for some youth. Furthermore, a goal of frequent experiencing of state mindfulness is to engender a more dispositional mindfulness over time (e.g., Brown \& Ryan, 2003; Carmody \& Baer, 2008) and thus the distinction between the two is not always clear. Finally, it is possible that those youth who report high mindfulness and depressive symptoms are identifying a greater awareness or focus on their present moment suffering, with little acceptance and not as an observer, and thus not actually experiencing mindfulness. Future qualitative or case studies might elucidate the process involved.

With regards to the exploratory examination of gender differences, gender was not correlated with mindfulness in either the NSSI or no-NSSI group and there were no gender differences in mindfulness in the group comparisons. The absence of gender differences in mindfulness is in line with previous research, which generally does not report gender differences in mindfulness in adolescents (e.g., Schonert-Reichl \& Lawlor, 2010; Tan \& Martin, 2012). However, a significant biserial correlation between gender and depressive symptoms was found only in the no-NSSI group, with females reporting higher depressive symptoms than males. Examination of the means by group and gender 
(Table 2) reveals that males and females in the NSSI group report similar high levels of depressive symptoms, whereas in the no-NSSI group females report slightly higher depressive symptoms than their male counterparts. This finding is not surprising as previous research using adolescent community samples has reported females indicating more depressive symptoms than males (Hankin \& Abramson, 2001; Nolen-Hoeksema, 2001), whereas no gender differences have been found in depressive symptoms amongst NSSI samples (e.g., Gandhi, Luyckx, Maitra, \& Claes, 2015). However, these results should be interpreted with caution as the group (NSSI, no-NSSI) by gender interaction effect was not significant suggesting the magnitude of the gender differences in the noNSSI versus in the NSSI groups were not substantially different.

As predicted, the NSSI group reported significantly more depressive symptoms and less mindfulness than the no-NSSI group. The higher depressive symptoms in the NSSI group is not unexpected in light of the substantial research documenting high depressive symptoms in youth who engage in NSSI (e.g., Garisch \& Wilson, 2015; Marshall et al., 2013; Peterson, Freedenthal, Sheldon, \& Andersen, 2008; Plener et al., 2015; You, Leung, \& Fu, 2012). However, the lower mindfulness in the NSSI group is a significant contribution to a very limited body of research. Specifically, as noted in the introduction, although there is evidence suggesting the importance of mindfulness to nonsuicidal self-injury in clinical populations (Wupperman et al., 2013) or deliberate selfharm in adults or youth (Lundh et al., 2007; Yusainy \& Lawrence, 2014), there is only one study of mindfulness as a correlate of NSSI in adolescents (Garisch \& Wilson, 2015). Thus, these results serve to provide additional support for the notion that amongst youth who engage in NSSI, as expected, reports of mindfulness are lower than for those who do 
not engage in self-injury. Yet, these results should be considered in conjunction with the finding that mindfulness functioned as a partial mediator between depressive symptoms and NSSI as discussed below.

The mediational model confirmed that mindfulness and depressive symptoms had a significant direct effect on the presence/absence of NSSI; however, there was also an indirect effect of depressive symptoms on NSSI through mindfulness. Specifically, when controlling for mindfulness the direct effect of depression on NSSI is lowered, although it remains significant. Thus, mindfulness partially explains the relationship between depressive symptoms and NSSI, providing the first empirical support for the protective role of mindfulness in NSSI for youth with depressive symptoms. The pattern of relationships found between depressive symptoms, mindfulness, and NSSI in the mediational model explicate common clinical assumptions about the benefits of mindfulness for self-injury in the presence of emotional distress, namely that mindfulness decreases rumination and increases acceptance and tolerance of difficult emotions in turn diminishing the need to self-injure (e.g., Gratz, 2007; Marks et al., 2010; Miller et al., 2009; Walsh, 2014). Despite these promising early results concerning the role of mindfulness in self-injury amongst youth, further research is needed to replicate the present findings as well as determine the potential differences in the differing role of dispositional mindfulness, as measured in the present study, versus a state of mindfulness elicited through treatment. In addition, future investigation should evaluate which facets of mindfulness (i.e., present moment awareness, acceptance and non-judgement) are contributing to the observed effect. Finally, particular attention is needed to better understand the subset of youth with self-injury who report high depressive symptoms and 
high mindfulness. Currently, causal inferences cannot be made without longitudinal or experimental design to examine the effect of changing state and dispositional mindfulness on the relationship between depressive symptoms and NSSI.

\section{Study Limitations}

Limitations of the present study include the overrepresentation of females in the NSSI sample and single time measurement of variables making causal inferences impossible. Furthermore, although the evaluation of non-suicidal self-injury was rigorous and a strength of the present study, consisting of both screening survey responses and individual interviews, self-injury could nevertheless have occurred any time in the past year and thus may or may not have been concurrent with the measures of mindfulness and depressive symptoms. 


\section{REFERENCES}

Arch, J. J., \& Craske, M. G. (2010). Laboratory stressors in clinically anxious and non-anxious individuals: The moderating role of mindfulness. Behavior Research and Therapy, 44(6), 495-505. doi:10.1016/j.brat.2010.02.005

Beauchemin, J., Hutchins, T. L., \& Patterson, F. (2008). Mindfulness meditation may lessen anxiety, promote social skills, and improve academic performance among adolescents with learning disabilities. Complementary Health Practice Review, 13(34), 34-45. doi:10.1177/1533210107311624

Beck, J., Beck, A., Jolly, J. B., \& Steer, R. (2005). Beck youth inventories for children and adolescents (2nd ed.). San Antonio, TX: PsyCorp.

Bracken-Minor, K. L., \& McDevitt-Murphy, M. E. (2014). Differences in features of nonsuicidal self-injury according to borderline personality disorder screening status. Archives of Suicide Research, 18(1), 88-103. doi:10.180/13811118.2013.809040

Branstrom, R., Duncan, L. G., \& Moskowitz, J. T. (2011). The association between dispositional mindfulness, psychological well-being, and perceived health in a Swedish population- 
based sample. British Journal of Health Psychology, 16(2), 300-316.

doi:10.1348/135910710x501683

Brickman, L. J., Ammerman, B. A., Look, A. E., Berman, M. E., \& McCloskey, M. S. (2014). The relationship between non-suicidal self-injury and borderline personality disorder symptoms in a college sample. Borderline Personality Disorder and Emotion Dysregulation, 1, 14. doi:10.1186/2051-6673-1-14

Brown, K. W., \& Ryan, R. M. (2003). The benefits of being present: Mindfulness and its role in psychological well-being. Journal of Personality and Social Psychology, 84(4), 822-848. doi:10.1037/0022-3514.84.4.822

Brown, K. W., Ryan, R. M., \& Creswell, J. D. (2007). Mindfulness: Theoretical foundations and evidence for its salutary effects. Psychological Inquiry, 18, 211-237. doi:10.1080/10478400701598298

Burke, C. A. (2010). Mindfulness-based approaches with children and adolescents: A preliminary review of current research in an emergent field. Journal of Child and Family Studies, 19(2), 133-144. doi:10.1007/s10926-009-9282-x

Carmody, J., \& Baer, R. (2008). Relationship between mindfulness practice and levels of mindfulness, medical and psychological symptoms and well-being in a mindfulnessbased stress reduction program. Journal of Behavioral Medicine, 31(1), 23-33. doi:10.1007/s10865-007-9130-7

Cash, M., \& Whittingham, K. (2010). What facets of mindfulness contribute to psychological well-being and depressive, anxious, and stress-related symptomatology? Mindfulness, 1, 177-182. doi:10.1007/s12671-010-0023-4 
Ciesla, J. A., Reilly, L. C., Dickson, K. S., Emanuel, A. S., \& Updegraff, J. A. (2012). Dispositional mindfulness moderates the effects of stress among adolescents: Rumination as a mediator. Journal of Clinical Child \& Adolescent Psychology, 41(6), 760-770. doi:10.1080/15374416.2012.698724

Frank, J. L., Jennings, P. A., \& Greenberg, M. T. (2013). Mindfulness-based interventions in school settings: An introduction to the special issue. Research in Human Development, 10(3), 205-210. doi:10.1080/15427609.2013.818480

Gandhi, A., Luyckx, K., Maitra, S., \& Claes, L. (2015). Non-suicidal self-injury and identity distress in Flemish adolescents: Exploring gender differences and mediational pathways. Personality and Individual Differences, 82, 215-220. doi:10.1016/j.paid.2015.03.031

Garisch, J. A., \& Wilson, M. S. (2015). Prevalence, correlates, and prospective predictors of nonsuicidal self-injury among New Zealand adolescents: Cross-sectional and longitudinal survey data. Child and Adolescent Psychiatry and Mental Health, 9, 28. doi:10.1186/s13034-015-0055-6

Gratz, K. L. (2007). Targeting emotion dysregulation in the treatment of self-injury. Journal of Clinical Psychology, 63(11), 1091-1103. doi:10.1002/jclp.20417

Greco, L. A., Baer, R. A., \& Smith, G. T. (2011). Assessing mindfulness in children and adolescents: Development and validation of the child and adolescent mindfulness measure (CAMM). Psychological Assessment, 23(3), 606-614. doi:10.1037/a0022819

Greenberg, M. T., \& Harris, A. R. (2012). Nurturing mindfulness in children and youth: Current state of research. Child Development Perspectives, 6(2), 161-166.

doi:10.1111/j.1750x8606.2011.00215 
Grossman, P., Niemann, L., Schmidt, S., \& Walach, H. (2004). Mindfulness-based stress reduction and health benefits: A meta-analysis. Journal of Psychosomatic Research, 57(1), 35-43. doi:10.1016/S0022-3999(03)00573-7

Hankin, B. L., \& Abela, J. R. Z. (2011). Non-suicidal self-injury in adolescence: Prospective rates and risk factors in a 2-year longitudinal study. Psychiatry Research, 186, 65-70. doi:10.1016/j.psychres.2010.07.056.

Hankin, B. L., \& Abramson, L. Y. (2001). Development of gender differences in depression: An elaborated cognitive vulnerability-transactional stress theory. Psychological Bulletin, 127(6), 773-796. doi:10.1037//0033-2909.127.6.773

Haw, C., Houston, K., Townsend, E., \& Hawton, K. (2002). Deliberate self harm patients with depressive disorders: Treatment and outcome. Journal of Affective Disorders, 70, 57-65. doi:10.1016/S0165-0327(01)00317-2

Heath, N., Toste, J., Nedecheva, T., \& Charlebois, A. (2008). An examination of non-suicidal self-injury among college students. Journal of Mental Health Counseling, 30(2), 137156. doi:10.17744/mehc.30.2.8p879p3443514678

Heath, N. L., \& Nixon, M. K. (2009). Assessment of non-suicidal self-injury in youth. In M. K. Nixon \& N. L. Heath (Eds.), Self-injury in youth: The essential guide to assessment and intervention (pp. 143-170). New York, NY: Routledge.

Heath, N. L., \& Ross, S. (2007). How I deal with stress. Unpublished instrument. Montreal, QC: McGill University. 
Hofmann, S. G., Sawyer, A. T., Witt, A. A., \& Oh, D. (2010). The effect of mindfulness-based therapy on anxiety and depression: A meta-analytic review. Journal of Consulting and Clinical Psychology, 78(2), 169-183. doi:10.1037/a0018555

Kabat-Zinn, J. (2003). Mindfulness-based interventions in context: Past, present, and future. Clinical Psychology: Science and Practice, 10(2), 144-156. doi:10.1093/clipsy/bpg016

Katz, L. Y., Cox, B. J., Gunasekara, S., \& Miller, A. L. (2004). Feasibility of dialectical behavior therapy for suicidal adolescent inpatients. Journal of the American Academy of Child \& Adolescent Psychiatry, 43(3), 276-282. doi:10.1097/01.chi.0000106854.88132.4f

Kaufman, J., Birmaher, B., Brent, D., Rao, U., Flynn, C., Moreci, P., ... Ryan, N. (1997). Schedule for affective disorders and schizophrenia for school age children, present and lifetime version (K-SADS-PL): Initial reliability and validity data. Journal of the American Academy of Child and Adolescent Psychiatry, 36, 980-988. doi:10.1097/00004583-199707000-00021

Laye-Gindhu, A., \& Schonert-Reichl, K. A. (2005). Non-suicidal self-harm among community adolescents: Understanding the "whats" and "whys" of self-harm. Journal of Youth and Adolescence, 34(5), 447-457. doi:10.1007/s10964-005-7262-z

Linehan, M. M., Comtois, K. A., Murray, A. M., Brown, M. Z., Gallop, R. J., Heard, H. L., ... Lindenboim, N. (2006). Two-year randomized controlled trial and follow-up of dialectical behavior therapy vs therapy by experts for suicidal behaviors and borderline personality disorder. Archives of General Psychiatry, 63(7), 757-766. doi:10.1001/archpsyc.63.7.757 
Lloyd, E. E., Kelley, M. L., \& Hope, T. (1997). Self-mutilation in a community sample of adolescents: Descriptive characteristics and provisional prevalence rates. Society for Behavioral Medicine annual meeting. New Orleans, LA.

Lloyd-Richardson, E. E., Perrine, N., Dierker, L., \& Kelley, M. L. (2007). Characteristics and functions of non-suicidal self-injury in a community sample of adolescents. Psychological Medicine, 37, 1183-1192. doi:10.1017/S003329170700027X

Lundh, L. G., Karim, J., \& Quilisch, E. (2007). Deliberate self-harm in 15-year-old adolescents: A pilot study with a modified version of the deliberate self-harm inventory. Journal of Psychology, 48, 33-41. doi:10.1111/j.1467-9450.2006.00567.x

Marks, A. D., Sobanski, D. J., \& Hine, D. W. (2010). Do dispositional rumination and/or mindfulness moderate the relationship between life hassles and psychological dysfunction in adolescents? Australian and New Zealand Journal of Psychiatry, 44(9), 831-838. doi:10.3109/00048674.2010.487478

Marshall, S. K., Tilton-Weaver, L. C., \& Stattin, H. (2013). Non-suicidal self-injury and depressive symptoms during middle adolescents: A longitudinal analysis. Journal of Youth and Adolescence, 42(8), 1234-1242. doi:10.1007/s10964-013-9919-3

Mendelson, T., Greenberg, M. T., Dariotis, J. K., Gould, L. F., Rhoades, B. L., \& Leaf, P. J. (2010). Feasibility and preliminary outcomes of a school-based mindfulness intervention for urban youth. Journal of Abnormal Child Psychology, 38(7), 985-994. doi:10.1007/s10802-010-9418-x

Miller, A. L. (1999). Dialectical behavior therapy: A new treatment approach for suicidal adolescents. American Journal of Psychotherapy, 53(3), 413-417. 
Miller, A. L., Muehlenkamp, J. J., \& Jacobson, C. M. (2009). Special issues in treating adolescent non-suicidal self-injury. In M. Nock (Eds.), Understanding non-suicidal selfinjury: Origins, assessment, and treatment (pp. 251-270). Washington, DC: American Psychological Association.

Miller, A. L., Wyman, S. E., Huppert, J. D., Glassman, S. L., \& Rathus, J. H. (2000). Analysis of behavioral skills utilized by suicidal adolescents receiving dialectical behavior therapy. Cognitive and Behavioral Practice, 7(2), 183-187. doi:10.1016/S1077-7229(00)80029-2

Muehlenkamp, J. J. (2006). Empirically supported treatments and general therapy guidelines for non-suicidal self-injury. Journal of Mental Health Counseling, 28(2), 166-185. doi:10.17744/mehc.28.2.6w61cut21xjdg3m7

Muehlenkamp, J. J., \& Gutierrez, P. M. (2007). Risk for suicide attempts among adolescents who engage in non-suicidal self-injury. Archives of Suicide Research, 11(1), 69-82. doi:10.1080/13811110600992902

Murphy, M. J., Mermelstein, L. C., Edwards, K. M., \& Gidycz, C. A. (2012). The benefits of dispositional mindfulness in physical health: A longitudinal study of female college students. Journal of American College Health, 60(5), 341-348. doi:10.1080/07448481.2011.629260

Nock, M. K. (2012). Future directions for the study of suicide and self-injury. Journal of Clinical Child \& Adolescent Psychology, 41(2), 255-259. doi:10.1080/15374416.2012.652001

Nock, M. K., Holmberg, E. V., Photos, V. I., \& Michel, B. D. (2007). Self-injurious thoughts and behaviors interviews: Development, reliability, and validity in an adolescent sample. Psychological Assessment, 19(3), 309-317. doi:10.1037/1040-3590.19.3.309 
Nolen-Hoeksema, S. (2001). Gender differences in depression. Current Directions in Psychological Science, 10(5), 173-176. doi:10.1111/1467-8721.00142

Peterson, J., Freedenthal, S., Sheldon, C., \& Andersen, R. (2008). Non-suicidal self-injury in adolescents. Psychiatry, 5(11), 20-26. doi:10.1037/e515372010-001

Plener, P. L., Schumacher, T. S., Munz, L. M., \& Groschwitz, R. C. (2015). The longitudinal course of non-suicidal self-injury and deliberate self-harm: A systematic review of the literature. Borderline Personality Disorder and Emotion Dysregulation, 2(1), 2. doi:10.1186/s40479-014-0024-3

Preacher, K. J., \& Hayes, A. F. (2004). SPSS and SAS procedures for estimating indirect effects in simple mediation models. Behavior Research Methods, Instruments, and Computers, 36, 717-731. doi:10.3758/BF03206553

Rasmussen, M. K., \& Pidgeon, A. M. (2011). The direct and indirect benefits of dispositional mindfulness on self-esteem and social anxiety. Anxiety, Stress \& Coping: An International Journal, 24(2), 227-233. doi:10.1080/106151806.2010.515681

Rathus, J. H., \& Miller, A. L. (2002). Dialectical behavior therapy adapted for suicidal adolescents. Suicide and Life-Threatening Behavior, 32(2), 146-157. doi:10.1521/suli.32.2.146.24399

Rodham, K., \& Hawton, K. (2009). Epidemiology and phenomenology of non-suicidal selfinjury. In M. K. Nock (Eds.), Understanding non-suicidal self-injury: Origins, assessment, and treatment (pp. 37-62). Washington, DC: American Psychological Association. 
Ross, S., \& Heath, N. (2002). A study of the frequency of self-mutilation in a community sample of adolescents. Journal of Youth and Adolescence, 31(1), 67-77. doi:10.1023/A:1014089117419

Sadeh, N., Londahl-Shaller, E. A., Piatigorsky, A., Fordwood, S., Stuart, B. K., McNiel, D. E., ... Yaeger, A. M. (2014). Functions of non-suicidal self-injury in adolescents and young adults with borderline personality disorder symptoms. Psychiatry Research, 216, $217-$ 222. doi:10.1016/j.psychres.2014.02.018

Schonert-Reichl, K. A., \& Lawlor, M. A. (2010). The effects of a mindfulness-based education program on pre- and early adolescents' well-being and social and emotional competence. Mindfulness, 1, 137-151. doi:10.1007/s12671-010-0011-8

Segal, Z. V., Williams, J. M. G., \& Teasdale, J. D. (2002). Mindfulness-based cognitive therapy for depression: A new approach to preventing relapse. New York, NY: Guilford.

Semple, R. J., Reid, E. F., \& Miller, L. (2005). Treating anxiety with mindfulness: An open trial of mindfulness training for anxious children. Journal of Cognitive Psychotherapy, 19(4), 379-392. doi:10.1891/jcop.2005.19.4.379

Stillman, C. M., Feldman, H., Wambach, C. G., Howard, J. H., \& Howard, D. V. (2014). Dispositional mindfulness is associated with reduced implicit learning. Consciousness and Cognition, 28, 141-150. doi:10.1016/j.concog.2014.07.002

Tacon, A., McComb, J., Caldera, Y., \& Randolph, P. (2003). Mindfulness meditation, anxiety reduction, and heart disease: A pilot study. Family and Community Health, 26(1), 25-33. doi:10.1097/00003727-200301000-00004 
Tan, L. B. G., \& Martin, G. (2012). Mind full or mindful: A report on mindfulness and psychological health in healthy adolescents. International Journal of Adolescence and Youth, 21, 64-74. doi:10.1080/02673843.2012.709174

Teasdale, J. D., Moore, R. G., Hayhurst, H., Pope, M., Williams, S., \& Segal, Z. V. (2002). Metacognitive awareness and prevention of relapse in depression: Empirical evidence. Journal of Consulting and Clinical Psychology, 70(2), 275-287. doi:10.1037//0022006X.70.2.275

Toste, J. R., Christie, M., \& Heath, N. L. (2011). How I deal with stress (HIDS): Evidence for the use of an NSSI screening questionnaire among young adults. Poster presented at the annual meeting of the international society for the study of self-injury (ISSS). New York, NY.

Wagner, E. E., Rathus, J. H., \& Miller, A. L. (2006). Mindfulness in dialectical behavior therapy (DBT) for adolescents. In R. A. Baer Mindfulness-based treatment approaches: Clinician's guide to evidence base and applications (pp. 167-190) Burlington, MA: Academic Press.

Walsh, B. W. (2012). Treating self-injury: A practical guide (2nd ed.). New York, NY: Guilford.

Whitlock, J. L., Powers, J. L., \& Eckenrode, J. (2006). The virtual cutting edge: The internet and adolescent self-injury. Developmental Psychology, 42(3), 407-417. doi:10.1037/00121649.42.3.000

Wupperman, P., Fickling, M., Klemanski, D. H., Berking, M., \& Whitman, J. B. (2013). Borderline personality features and harmful dysregulated behavior: The meditational 
effect of mindfulness. Journal of Clinical Psychology, 69(9), 903-911.

doi: $10.1002 / j c l p .21969$

You, J., Leung, F., \& Fu, K. (2012). Exploring the reciprocal relations between non-suicidal selfinjury, negative emotions and relationship problems in Chinese adolescents: A longitudinal cross-lag study. Journal of Abnormal Child Psychology, 40(5), 829-836. doi:10.1007/s10802-011-9597-0

Yusainy, C., \& Lawrence, C. (2014). Relating mindfulness and self-control to harm to the self and to others. Personality and Individual Differences, 64, 78-83. doi:10.1016/j.paid.2014.02.015 
FIGURE $1(a, b)$ Scatterplots showing the association between mindfulness and depressive symptoms as a function of (a) NSSI Group and (b) No NSSI group. The solid line represents the linear trend. The dashed line represents clinical cutoff for depressive symptoms as indicated by the BDI-Y. Participants within the circle in (a) reported high mindfulness and high depression.

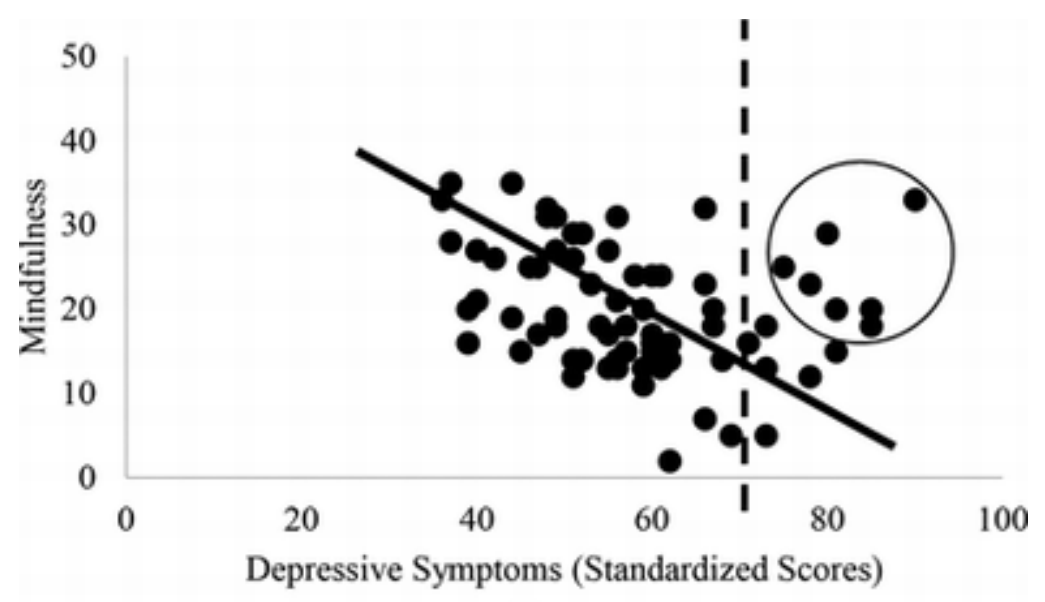

(a)

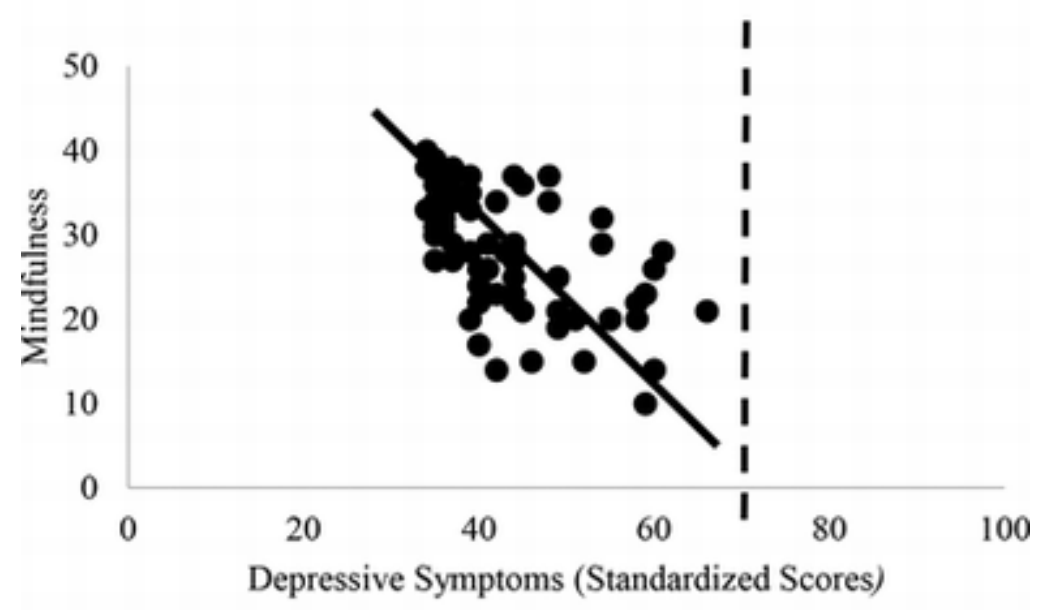

(b) 


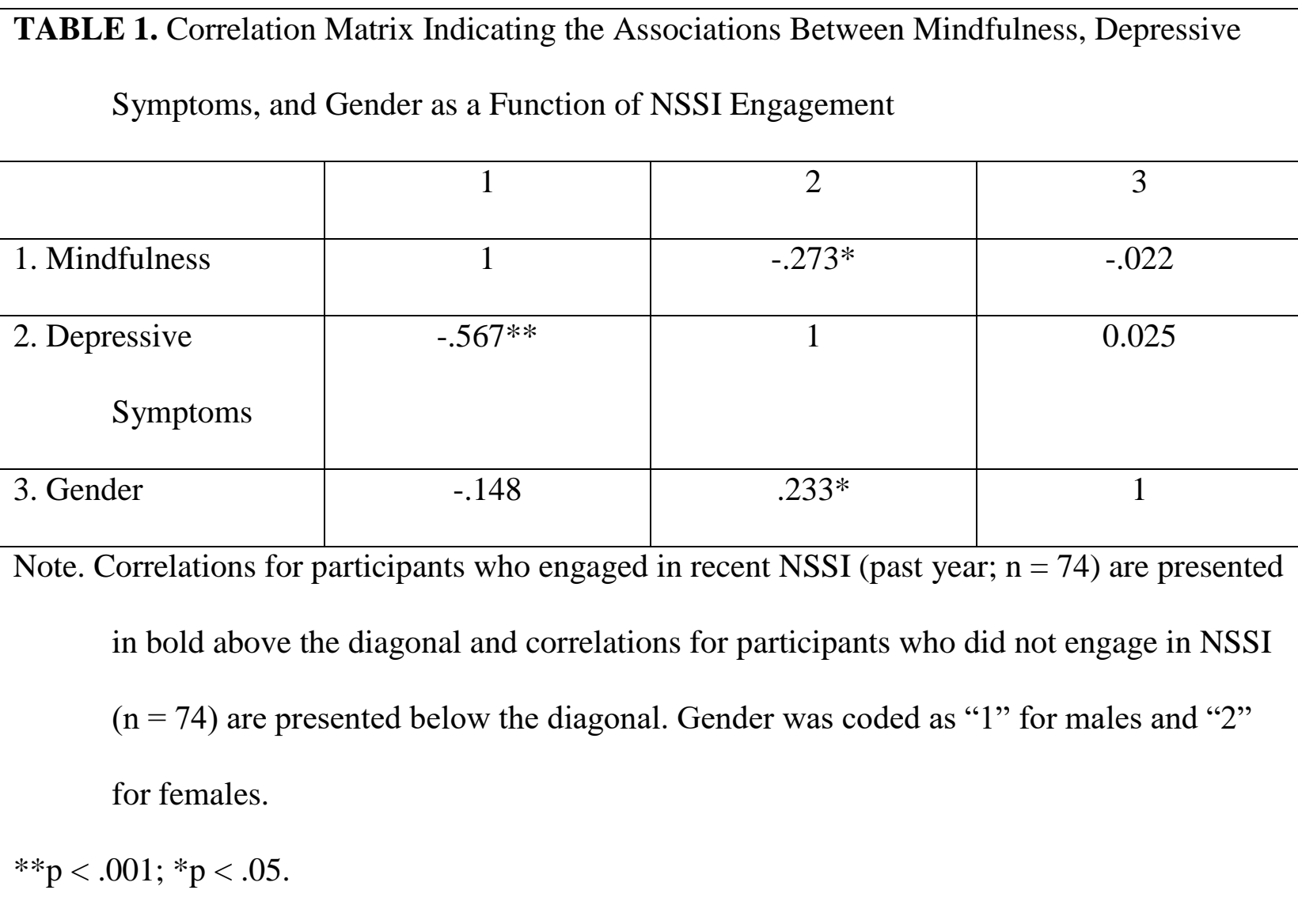

\begin{tabular}{|c|c|c|c|c|c|c|}
\hline \multicolumn{7}{|c|}{ NSSI and No-NSSI Groups by Gender } \\
\hline & \multicolumn{3}{|c|}{ No-NSSI } & \multicolumn{3}{|c|}{ NSSI } \\
\hline & Male & Female & Total & Male & Female & Total \\
\hline & M (SD) & M (SD) & M (SD) & M (SD) & M (SD) & M (SD) \\
\hline $\mathrm{n}$ & 12 & 62 & 74 & 12 & 62 & 74 \\
\hline Depressive & 38.75 & 43.97 & 43.12 & 57.67 & 58.52 & 58.38 \\
\hline Sympt & $(6$. & $(8$. & $(8$. & (17. & (11. & $(12$. \\
\hline oms & & & & 14) & 96) & 80) \\
\hline
\end{tabular}




\begin{tabular}{|l|r|r|r|r|r|r|}
\hline & 68 & 38 & 32 & & & \\
\hline Mindfulness & 30.42 & 27.50 & 27.97 & 20.42 & 19.98 & 20.05 \\
& $(5$. & $(7$. & $(7$. & $(8.6$ & $(7.1$ & $(7.3$ \\
& 93 & 49 & 31 & $5)$ & $6)$ & $5)$ \\
& & & & & & \\
\hline
\end{tabular}

FIGURE 2 Model depicting the association between depressive symptoms, NSSI status and the mediational role of mindfulness. ${ }^{*} \mathrm{p}<.05 * * \mathrm{p}<.01 * * * \mathrm{p}<.001$.

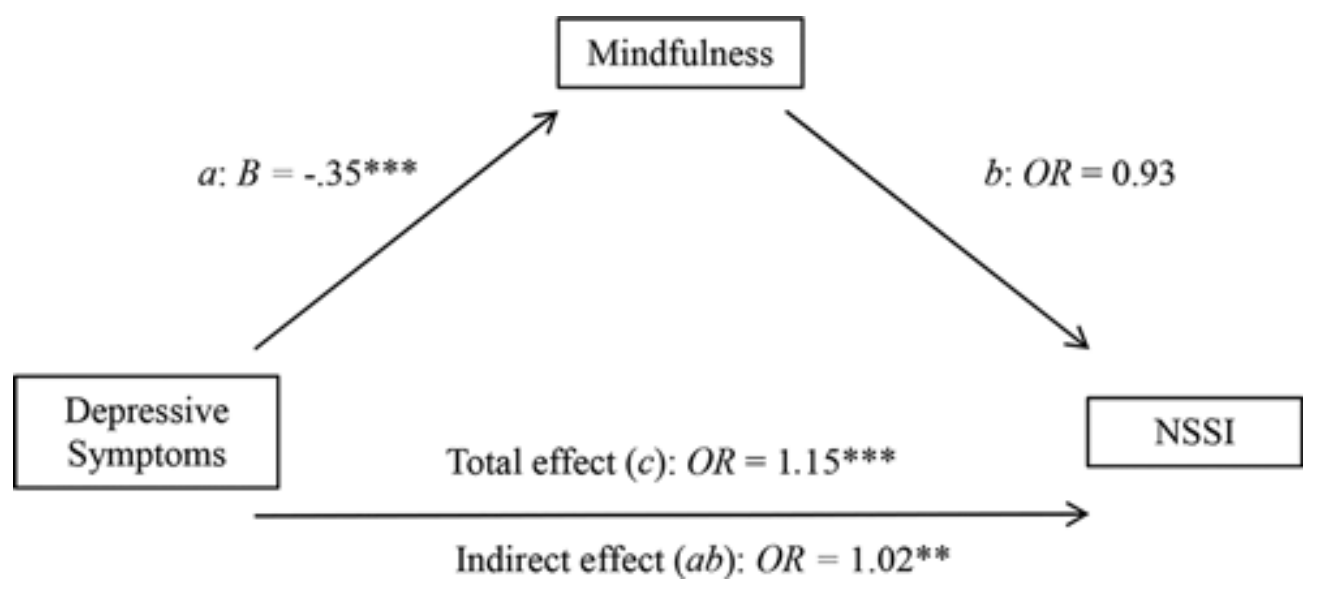

